\title{
BREEDING FHB-RESISTANT SOFT WINTER WHEAT: PROGRESS AND PROSPECTS
}

Gina BROWN-GUEDIRA ${ }^{1}$ - Carl GRIFFEY ${ }^{2}$-Fred KOLB ${ }^{3}$ - Anne MCKENDRY ${ }^{4}-J$. Paul MURPHY ${ }^{5}$ - David VAN SANFORD ${ }^{6}$

${ }^{1}$ Eastern Regional Small Grains Genotyping Lab, USDA-ARS, Raleigh, NC 27695-7620, email: gina brown-guedira@ncsu.edu

${ }^{2}$ Department of Crop and Soil Environmental Sciences, Virginia Tech, Blacksburg, VA 24061, email: cgriffey@vt.edu

${ }_{3}^{3}$ Department of Crop Sciences, University of Illinois, Urbana, IL 61801, email: f-kolb@illinois.edu ${ }^{4}$ Division of Plant Sciences, University of Missouri, Columbia MO USA 65211, email: mckendrya@missouri.edu

${ }^{5}$ Department of Crop Science, North Carolina State University, Raleigh, NC 27695, email: paul_murphy@ncsu.edu

${ }^{6}$ Department of Plant and Soil Sciences, University of Kentucky, Lexington, KY 40546, email: dvs@uky.edu

Abstract: Soft winter wheat (Triticum aestivum L.) breeding programs in the US have used two general approaches to developing FHB-resistant cultivars: 1) incorporation of $F h b 1$ plus other minor QTL from Asian wheat cultivars and their derivatives and 2) reliance on resistance native to the soft winter wheat gene pool. Although each approach has shown some success, it is believed that the two must be integrated to develop the highest levels of resistance. The most favorable scenario for integration is the incorporation of Fhb1 into adapted material with good native resistance, high yield and test weight, and superior milling and baking quality.

Keywords: Fusarium head blight, wheat breeding, resistance

\section{Introduction}

The 1990's were a decade of serious scab epidemics in certain wheat producing areas of the U.S. Following the devastating 1993 head scab epidemic in the Northern Great Plains a very severe epidemic hit the northern Corn Belt in 1996, with the result that low deoxynivalenol (DON) wheat was almost impossible to find (Rudd et al. 2001). Soon afterwards, under the auspices of the US Wheat and Barley Scab Initiative (USWBSI; http://www.scabusa.org) researchers launched a massive effort to reduce the economic impact of FHB on the wheat and barley (Hordeum vulgare L.) industries in the United States. Development of resistant cultivars has been a primary thrust of this research effort. In the ensuing decade, public and private breeding programs have targeted FHB resistance as a "must have" trait. Through the USWBSI, soft winter wheat breeding programs have collaborated through participation in uniform screening nurseries, sharing germplasm, and data. Individual breeding programs, however, have chosen their own approach to breeding resistant cultivars. Breeders have followed two pathways towards resistance: 1) incorporation of Fhb1 plus other minor QTL and 2) utilization of resistance genes native to the soft winter wheat gene pool.

\section{Materials and Methods}

\section{Use of Fhb1 resistance}

Most, if not all, of the soft wheat breeding programs have used $F h b 1$ in their variety development efforts. Backcross and doubled haploid, as well as traditional breeding methods have been used in conjunction with phenotypic and marker assisted selection (MAS) in variety development efforts. A majority of the breeding programs are 
currently using MAS either in their own labs or in cooperation with the USDA-ARS Eastern Regional Small Grains Genotyping Lab (Van Sanford et al. 2001) in characterization and selection of parents and pure lines, backcrossing, and population enrichment for Fhb1 (Bonnett et al. 2005). In addition to Fhb1, programs are incorporating and pyramiding FHB resistance QTL located on wheat chromosomes 1B, 2B, 2D, 3A, 3BSc, 4B, 5A, and 6B as well as from wheat relatives including $Q$ fhs.ndsu$3 A S$ from $T$. dicoccoides and $Q$ fhs.pur-7EL from tall wheatgrass.

\section{Use of native resistance}

All of the soft winter wheat breeding programs routinely screen their own breeding lines, adapted cultivars, and colleagues' breeding lines available through an array of cooperative nurseries in search of native scab resistance. Since this resistance has usually not been haplotyped, this effort requires very extensive phenotypic evaluation. Typically, this is done in mist-irrigated screening nurseries and in some, but not all cases, through point inoculation in the greenhouse. Inoculum in the field nurseries is in the form of multiple conidial spray applications or grain spawn in which scabby maize (Zea mays L.) or wheat is spread throughout the nursery prior to flowering. Promising lines are evaluated a second and third time, in some cases through the USWBSI uniform screening nurseries at multiple locations. If the FHB phenotype is stable, these lines are used as parents in single or three-way crosses with other adapted genotypes that have acceptable agronomic, disease, and quality profiles. The goal of this effort is to incrementally add favorable scab resistance alleles to breeding material that is on track for cultivar release.

\section{FHB traits and resistance}

Initially, most programs focused on Type II resistance, and this trait continues to receive considerable attention, whether the emphasis is on MAS for Fhbl or point inoculation of putative native resistant types in the greenhouse. Type I resistance, although present in cultivars such as Goldfield (H. Ohm, personal communication) and Truman (McKendry et al. 2005), remains largely elusive. In recent years, because of economic penalties and food safety concerns associated with high DON levels, the emphasis has shifted to reducing DON and Fusarium damaged kernels (FDK). Although independent resistance to DON has been postulated (Mesterhazy 1995), most soft winter wheat breeders continue to concentrate on reducing FHB in the crop. Thus the usual array of traits measured in field nurseries includes incidence, severity, FHB index (incidence $\mathrm{x}$ severity), FDK, ISK $(0.3 *$ Incidence $+0.3 *$ Severity $+0.4 *$ Fusarium Damaged Kernels) and DON

\section{Results and Discussion}

Cultivars released with Fhb1 resistance

To date only a few commercial soft wheat cultivars possessing $F h b 1$ have been released and include INW0411 and Pioneer Brands 25R18, 25R42, and 25R51. Several additional programs likely will release cultivars with $F h b 1$ within the next five years. While initial efforts to transfer $F h b 1$ into adapted wheat backgrounds were hindered by the lack of diagnostic markers that were reliable in diverse germplasm, this problem has 
been largely overcome. Many programs have developed improved soft wheat germplasm lines possessing $F h b 1$, and several programs have, or soon will register, germplasm lines such as OH904, VA04W-433, and VA04W-563. The paucity of commercial soft wheat cultivars with $F h b 1$ released to date is primarily a reflection of the difficulties in breaking unfavorable linkages between FHB resistance and low yield, shattering, poor quality, and susceptibility to other major diseases including leaf rust (Puccinia triticina Eriks.), stripe rust (Puccinia striiformis Westend.), wheat soil borne mosaic virus, and glume blotch [Stagonospora nodorum (Berk.) Castellani \& E.G.Germano]. In a backcrossing program one can eliminate most of the donor parent genes except for $F h b 1$ and other QTL which have been selected through MAS. In this case the recurrent parent itself needs to have a very good level of FHB resistance because one has selected against any other FHB resistance QTL in the donor parent. A fundamental problem is that FHB resistance is a quantitatively inherited trait, and although $F h b 1$ is a major locus, it is not sufficient to confer the desired level of scab resistance. In spring wheat, for example, Pumphrey et al. (2007) in a comparison of near isogenic lines, estimated that $F h b 1$ conferred an average reduction of $27 \%$ of FHB damaged kernels.

Cultivars released with native resistance

Numerous SRW wheat cultivars with native FHB resistance have been released including Freedom, COKER 9474, Foster, Pembroke, Patton, Roane, Goldfield, McCormick, Tribute, NC-Neuse, INW0304, IL94-1653, IL00-8061, INW0411, NY88046-8138, COKER 9511, and WestBred X00-1079 among others (Griffey 2005). The most striking success has been achieved by the Missouri breeding program, which has to its credit the release of 'Ernie', 'Truman' and 'Bess' (McKendry et al. 1995; 2005 ; 2007). Scab resistance alleles have been selected by the Missouri breeding program through selection for high yield and test weight under disease pressure, (McKendry 2008). Since all breeders select for increased yield and test weight, and usually without a concomitant increase in FHB resistance, this suggests that the Missouri environment is unusual.

One of the biggest impediments to identification of native resistance is the difficulty in replicating favorable selection environments. This fact underscores the importance of phenotyping, and in turn, the difficulty in creating an inoculated nursery environment in which the expression of these favorable alleles can be perceived. The inherent problems associated with misted field nurseries include differences in plant maturity, height and architecture, but the biggest problem may be that of excessive disease pressure. In a year in which natural conditions favor a scab epidemic, disease levels in a misted scab nursery may be so high that it is impossible to recognize the intermediate levels of resistance conferred by these native alleles. Late development of the disease in a misted nursery may result in underestimates of severity that are not highly correlated with FDK and DON concentration. In addition, Stagonospora nodorum infection on heads, promoted by daily misting, reduces the accuracy of phenotyping for FHB. These phenotyping issues are critical if we are to be successful in utilizing native resistance genes in concert with Fhb1 and other QTL. While much attention has been directed to 
the need to develop diagnostic markers, effective phenotyping protocols are at least as important for breeding success

\section{Long Term Outlook}

The importance of native resistance in the soft red winter wheat gene pool was initially underestimated by breeders who followed the lead of their colleagues working with the Hard Spring class, where native resistance is absent. Work is underway to identify resistance QTL controlling native resistance in the soft winter wheat gene pool and these loci will play important roles in MAS in the coming decade. High levels of FHB resistance will likely result from the combined effects of native and exotic resistances from within the cultivated gene pool. Because additive effects are the predominant form of gene action for most quantitative traits, it is reasonable to expect the same to be true for FHB resistance. Efforts will continue, to a much lesser extent, on sources of resistance from wheat relatives, but there is evidence from the Purdue program that exotic sources, such as wheatgrass, also act in an additive fashion with primary genepool sources such as Fhb1.

There have been few released cultivars that are known to have pyramided QTL for FHB resistance including INW0411 (Fhb1, 1B, and 3A), INW0731 and INW0801 (both with $1 \mathrm{~B}$ and $3 \mathrm{~A}$ ). In general, the limitations to pyramiding resistance QTL other than $F h b 1$ have been: lack of verification of QTL effects in diverse backgrounds, lack of fine mapping of large regions showing QTL effects and scarcity of markers that are diagnostic in diverse germplasm. Most of these QTL have $\mathrm{R}^{2}$ values lower than that reported for $F h b 1$. However, these QTL can be combined to provide resistance given there is germplasm with good resistance that does not have Fhb1. McCartney et al. (2007) demonstrated the effects of Fhb3 and the chromosome 5A region in their accelerated backcrossing project. These are additional candidate regions for pyramiding. Recent haplotyping of large numbers of FHB-resistant SRW experimental lines indicated that lines are available with the 5AS and 6B QTL regions introgressed from Chinese sources, as well as the 3BS region from Ernie. There is a real need for identification and validation of QTL in SRW germplasm, fine-mapping to narrow down the QTL regions and development of diagnostic markers.

\section{Conclusions}

This decade's efforts represent the first round of parent building and cultivar release. Breeding programs throughout the soft wheat region have built a high frequency of FHB resistance alleles into their cultivar development populations. The numbers of resistant entries in the Uniform FHB Nurseries are significantly increased over the past five years and high-productivity, advanced lines with good overall agronomic and enduse quality combined with FHB resistance are appearing in these nurseries at greater frequencies. But FHB resistance is a low heritability trait, just like grain yield. There is no silver bullet, although progress has been steady. The incremental nature of this progress merely underscores the intransigence of the disease. In fact, prospects for the mid- and long-term are good. There is some very good FHB resistance in the soft red 
winter wheat gene pool. While introgression of $F h b 1$ can build on this resistance, the likelihood of success is much greater if this important QTL is incorporated into backgrounds that already have strong resistance. Although MAS will become more commonplace, particularly as markers for native QTL become available, the importance of careful, accurate phenotypic evaluation is paramount.

\section{References}

Bonnett, D.G., Rebetzke, G.J., Spielmeyer, W. 2005. Strategies for efficient implementation of molecular markers in wheat breeding. Mol. Breeding 15:75-85.

Griffey, C.A. 2005. Identification and Incorporation of FHB Resistance in Winter Wheat: An Overview of Progress and Strategies. In: Canty, S.M., Boring, T., Wardwell, J., Siler, L., Ward, R. (eds), Proceedings of the 2005 National Fusarium Head Blight Forum; 2005 Dec. 11-13; Milwaukee, WI. East Lansing: Michigan State University, pp. 31-34.

McCartney, C.A., Somers, D.J., Fedak, G., DePauw, R.M., Thomas, J., Fox, S.L., Humphreys, D.G., Lukow, O., Savard, M.E., McCallum, B.D., Gilbert, J., Cao, W. 2007. The evaluation of FHB resistance QTLs introgressed into elite Canadian spring wheat germplasm. Molecular Breeding 20:209-221.

McKendry, Anne. 2008. Native Resistance: An Essential Building Block for Accelerating the Development of Scab Resistant Soft Red Winter Wheat. In: Proceedings of the 3rd International Symposium on Fusarium Head Blight; 2008 Sept. 1-5; Szeged, Hungary, Cereal Research Communications (IF: 1.037).

McKendry, A.L., Berg, J.E., Tague, D.N., Kephart, K.D. 1995. Registration of 'Ernie' Wheat. Crop Sci. 35:1513.

McKendry, A.L., Tague, D.N., Wright, R.L., Tremain, J.A., Conley, S.P. 2005. Registration of 'Truman' Wheat. Crop Sci. 45:421-423.

McKendry, A.L., Tague, D.N., Wright, R.L., Tremain, J.A. 2007. Registration of 'Bess' Wheat. Journal of Plant Registrations 1:21-23.

Mesterhazy, A. 1995. Types and components of resistance to Fusarium head blight of wheat. Plant Breeding 114:377-386.

Pumphrey, M.O., Bernardo, R., Anderson, J.A. 2007. Validating the Fhb1 QTL for Fusarium head blight resistance in near-isogenic wheat lines developed from breeding populations. Crop Sci. 47:200-206.

Rudd, J.C., Horsley, R.D., McKendry, A.L., Elias, E.M. 2001. Host plant resistance genes for Fusarium head blight: sources, mechanisms, and utility in conventional breeding systems. Crop Sci. 41:620-627.

Van Sanford, D., Anderson, J., Campbell, K., Costa, J., Cregan, P., Griffey, C., Hayes, P., Ward, R. 2001. Discovery and deployment of molecular markers linked to Fusarium head blight resistance: An integrated system for wheat and barley. Crop Sci. 41:638-644. 